\title{
TIPOS DE TIEMPO Y ADMISIONES HOSPITALARIAS ASOCIADAS A LA GRIPE EN GALICIA EN EL PERIODO 2003-2013
}

\author{
Pablo FDEZ-ARROYABE ${ }^{1}$, Dominic ROYÉ ${ }^{1}$, Alberto MARTI-EZPELETA ${ }^{2}$ \\ ${ }^{1}$ Universidad de Cantabria. Depto. de Geografia. GeoBioMet Group. \\ ${ }^{2}$ Universidad de Santiago de Compostela Depto. de Geografia. GeoBiomet Group. \\ fernandhp@unican.es, dominic.roye@unican.es, alberto.marti@usc.es
}

\section{RESUMEN}

El objetivo del trabajo es estudiar la relación entre los ingresos en hospitales públicos asociados a la gripe y los tipos de tiempo en la Comunidad Autónoma de Galicia para el periodo 2003-2013. Para ello se emplean dos clasificaciones diferentes. Con la primera de ámbito peninsular se definen los riesgos relativos asociados a cada categoría en relación a las admisiones hospitalarias asociadas al código gripe. Con la segunda centrada en Galicia se interpretan las situaciones sinópticas en relación a la evolución de las tasas de gripe y las admisiones hospitalarias desde un enfoque biometeorológico. Los resultados obtenidos indican que el riesgo de ingresos asociados a la gripe en Galicia es mayor con tipos de circulación atmosférica propios del $3^{\circ}$ y $4^{\circ}$ cuadrante cuando acontecen precedidos de situaciones de primer y segundo cuadrante. Algunos aspectos sociales son también importantes en explicar los incrementos de ingresos hospitalarios.

Palabras clave: Influenza, tipo de tiempo, riesgo relativo, admisiones hospitalarias.

\begin{abstract}
The aim of this work is to study the relationship between influenza related public hospitals admissions and circulation weather types in the Autonomous Community of Galicia (Northern Spain) for the period 2003-2013. Initially a CWT for the Iberian Peninsula is used to model the relative risk of each category in relation to admissions. Moreover a second much more specific classification, focused on Galicia region is considered in order to validate the theory related to the link between a specific spell in weather types can facilitate the spreading of the influenza epidemic and consequently increase the number of influenza related hospital admissions in Galicia. The results indicate that the risk of hospital admissions associated with influenza in Galicia is higher with types of atmospheric circulation of the 3rd and 4th quadrant preceded by first and second quadrant weather types. Some social aspects are also important in explaining increases in influenza-relate hospital admissions.
\end{abstract}

Key words: Influenza, weather types, relative risk, crowding, hospital admissions.

\section{INTRODUCCIÓN}

El riesgo de contraer gripe está asociado a múltiples factores, desde aspectos microbiológicos, epidemiológicos, socioculturales, sanitarios, geográficos o inmuno- 
lógicos, hasta los ambientales, siendo muy compleja la modelización de este fenómeno. La estacionalidad de la enfermedad en latitudes templadas está asociada a la estacionalidad climática lo que pone de manifiesto la relevancia meteorológica de las infecciones respiratorias agudas (IRA) en general y de la influenza en particular. Desde un punto de vista microbiológico, se ha constatado a través de la experimentación en laboratorio que variables como la temperatura y la humedad son importantes en relación a la probabilidad de infección (Lowen et al., 2006). La supervivencia del virus y su estacionalidad han sido estudiadas en zonas templadas (Tamerius et al. 2010, 2013; Bloom-Feshbach et al., 2013) y también su relación con diferentes variables meteorológicas. Por ejemplo, la radiación solar (Jensen, 1964) y el aumento de la temperatura actúan como factores limitantes de la supervivencia del microorganismo (Polozov et al., 2008; Fdez-Arroyabe, 2012). Por el contrario, la supervivencia del mismo aumenta cuando desciende la humedad en el aerosol y la superficie en donde se encuentra (Schaffer et al. 1976; Shaman y Kohn, 2009). La transmisión del virus es más eficiente con valores bajos de humedad relativa para una misma temperatura (Lowen et al. 2007 y 2008). La precipitación se ha vinculado a una reducción en la tasa de contagio entre los niños en los colegios según Mossong et al. (2008) habiéndose constatado que el virus puede viajar en pasajeros de aviones de unos lugares a otros (Moser et al. 1979).

La complejidad multifactorial y no lineal de las relaciones entre las variables ambientales, sociales y, la difusión de una enfermedad infecciosa como la influenza es elevada. Mucho más si consideramos que la atmósfera está lejos de ser un entorno controlado lo que implica una gran dificultad a la hora de encontrar respuestas. Las aproximaciones habituales parten del estudio de los tipos de tiempo y de la circulación atmosférica que afectan a la zona estudiada. Se han desarrollado clasificaciones climáticas de todo tipo (Lee y Sheridan, 2015) basadas en patrones de circulación atmosférica (CPC), muchas veces expresados con mapas, o en el análisis de las propiedades físicas de las masas de aire (WTC) como Kalkstein y Sheridan (2004) con el fin de asociar los tipos y las características física de las masas de aire a situaciones anómalas de mortalidad y morbilidad en distintos grupos de enfermedades. Desde la Biometeorología se han planteado métodos de base ecológica que consisten en identificar las situaciones de cambio en la atmósfera que propician la expansión del vector de transmisión de la enfermedad o que reducen la vulnerabilidad del receptor a causa del distrés biometeorológico (Fdez-Arroyabe, 2004) generado por esos cambios. Lo que es habitual es que las epidemias de gripe impliquen aumentos en los ingresos hospitalarios con sus correspondientes costes sociales y económicos. El número de casos de gripe diagnosticados en Galicia para el periodo 2003-13 fue de 113.957 según los registros oficiales de Enfermedades de Declaración Obligatoria (EDO) lo que supone una media anual de 10.359 casos. El número de admisiones hospitalarias (AH) asociadas a la gripe para ese periodo fue de 5.830 personas. Según el estudio de Badia et al. (2006) realizado sobre una muestra longitudinal y multicéntrica de 662 individuos en España los costes hospitalarios y los costes de absentismo laboral asociados a la gripe representan la mayor carga económica de este fenómeno, siendo el coste muy superior entre los pacientes considerados grupos de riesgo y especialmente en los casos crónicos. De acuerdo a este trabajo, la epidemia de gripe en España durante el 
año previo al periodo de estudio aquí planteado, para una incidencia media estimada del 7,9\%, supuso un coste de 1.036 millones de euros.

\subsection{Hipótesis de trabajo}

El riesgo de ser ingresado en un hospital público en Galicia durante la fase de desarrollo de las epidemias gripales está relacionado con el tipo de tiempo existente los días previos a la hospitalización y su secuenciación. Ciertos tipos de tiempo resultan más favorables al desarrollo y la expansión de las epidemias gripales y en consecuencia al aumento de las AH en esta comunidad autónoma, De ser esto así, estaríamos confirmando que las tesis validadas en entornos controlados, pueden ser, en cierto modo, extrapolables al ámbito atmosférico, en este caso para la región de Galicia.

El fin último del uso integrado de estos métodos de trabajo consiste en poder definir las secuencias atmosféricas propicias para el desarrollo de la enfermedad en Galicia con el fin de poder elaborar un sistema de alerta que anticipe el nivel de riesgo biometeorológico en materia de epidemias gripales y reduzca los efectos negativos de estos impacto en cuanto a las admisiones hospitalarias de esta Comunidad.

\subsection{Fuentes de datos}

Las fuentes de datos empleadas en este trabajo han sido meteorológicas y médicas. Para la elaboración de las categorías de tipos de circulación atmosférica han sido empleados los datos de presión atmosférica de la superficie del National Center for Atmospheric Research (NCAR) obtenidos por reanálisis. En segundo lugar, los datos diarios relativos a los ingresos urgentes en los hospitales clínicos públicos de Galicia para el periodo 2003-2013 se han obtenido de los registros hospitalarios del Conjunto Mínimo Básico de Datos (CMBD) a través del Ministerio de Sanidad. De los registros de hospitalizaciones han sido seleccionadas las causas patológicas, diagnóstico principal o secundario, con la clasificación de gripe 487 y 488 (CIE-9). Finalmente, los registros semanales de casos y tasas de gripe por cien mil habitantes para el mismo periodo de estudio han sido facilitados por la Red Nacional de Vigilancia Epidemiológica y provienen del sistema de registro EDO, habiendo sido facilitados por el Instituto Nacional de Epidemiología.

\section{MÉTODOS}

\subsection{Modelización estadística}

En primer lugar, se plantea la validación estadística de la hipótesis de trabajo a partir de la modelización estadística de los riesgos relativos. Existen numerosos métodos en la epidemiologia ambiental para cuantificar los efectos de riesgo en la salud humana (series temporales, estudios de cohorte, diseños de cruce, etc.) (Ye et al., 2012; Peng et al., 2008). En este estudio se aplica un modelo aditivo generalizado (GAM) (Hastie y Tibshirani, 1990) a series temporales para cuantificar el riesgo relativo (RR) de la asociación entre los tipos de circulación atmosférica (CWT) y los ingresos hospitalarios por gripe. Para el proceso de ajuste del GAM se calculan los retardos absolutos entre 1-7 días, con el objetivo de tener en cuenta posibles efectos retardados. Además de los CWT, que son introducidos en el modelo como una variable factor, se 
controlan la estacionalidad y la tendencia con la enumeración diaria de toda la serie temporal de forma cuadrada (Analitis et al., 2008). Se establecen 3 grados de libertad por cada año para la función de suavizado (Maclure, 1991). Los resultados de RR se representan con intervalos de confianza del 95\% (IC95). El RR indica la probabilidad de un ingreso hospitalario en relación a la variable de exposición. En la interpretación del RR se debe saber que éste siempre es mayor que 0 . Un valor menor de 1 se puede entender como protector, por ejemplo, un RR de 0,63 se traduce en una reducción del riesgo del 37\% (1-RR*100) con respecto al de referencia. En el caso contrario, si el RR es mayor que 1, se entiende como aumento de riesgo. Por ejemplo, un RR de 1,78 se traduce en un incremento del riesgo del $78 \%(\mathrm{RR}-1 * 100)$ con respecto al de referencia. Todos los modelos, el análisis estadístico, así como los resultados gráficos se realizan con el entorno de software libre R, versión 3.3. Los modelos aditivos generalizados se han estimado a través del paquete $\{m g c v\}$, versión 1.8-12.

\subsection{Enfoque biometeorológico}

Para complementar el estudio de la posible relación de los tipos de tiempo con los ingresos por gripe en los hospitales públicos gallegos y con las tasas de gripe y sus variaciones semanales, hemos analizado la frecuencia y las secuencias de las diferentes situaciones atmosféricas en Galicia durante los días previos a los periodos de expansión de la epidemia, a los mayores incrementos semanales de EDO y a los mayores incrementos de ingresos hospitalarios por gripe. Para ello hemos utilizado una clasificación de situaciones atmosféricas adaptada a la zona de estudio, poniendo el centro de los puntos para dicha clasificación en Galicia. El procedimiento empleado fue desarrollado por Lorenzo et al. (2008) para Galicia, y por Trigo y DaCamara (2000) para Portugal, adaptándolo de Jenkinson \& Collison (1977) y Jones (1993). Se calcularon la dirección y la vorticidad de los flujos geostróficos diarios para el periodo 2003-2013. Dicha clasificación ya ha sido empleada en un estudio anterior que analiza la relación entre los ingresos hospitalarios diarios en Galicia por causas respiratorias y las situaciones atmosféricas (Royé et al., 2015). Igualmente, se ha propuesto una nueva aproximación mediante la definición de las situaciones más contrastantes consideradas en la hipótesis de trabajo que se corresponden, por un lado, con las situaciones correspondientes al $1^{\circ}$ y $2^{\circ}$ cuadrantes, $\left(0^{\circ}\right.$ a $\left.180^{\circ}\right)$ con situaciones de N, NE, E, SE, que en Galicia suelen dar lugar a tipos de tiempo secos, con poca precipitación, más fríos y con menos humedad, y por otro las propias de los tipos del $3^{\circ}$ y $4^{\circ}$ cuadrantes $\left(180^{\circ}\right.$ a $360^{\circ}$ relativas a los tipos de circulación del $\left.\mathrm{S}, \mathrm{SW}, \mathrm{W}, \mathrm{NW}\right)$ que dan situaciones más inestables, con mayores precipitaciones en invierno, con mucha mayor humedad y con temperaturas más suaves para la época.

\section{RESULTADOS Y DISCUSION}

Las admisiones hospitalarias en periodo epidémico se encuentran condicionadas en cierto modo por el número de casos de gripe de cada semana epidemiológica. La variación semanal de la tasa gripal se encuentra correlacionada con las $\mathrm{AH}$ con un coeficiente superior al 0,5 según Pearson. Esta relación puede aumentar si consideráramos los subgrupos de mayor riesgo (enfermos crónicos y mayores de 65 años), que 
como ya hemos señalado previamente representan los mayores impactos en cuanto a costes económicos.

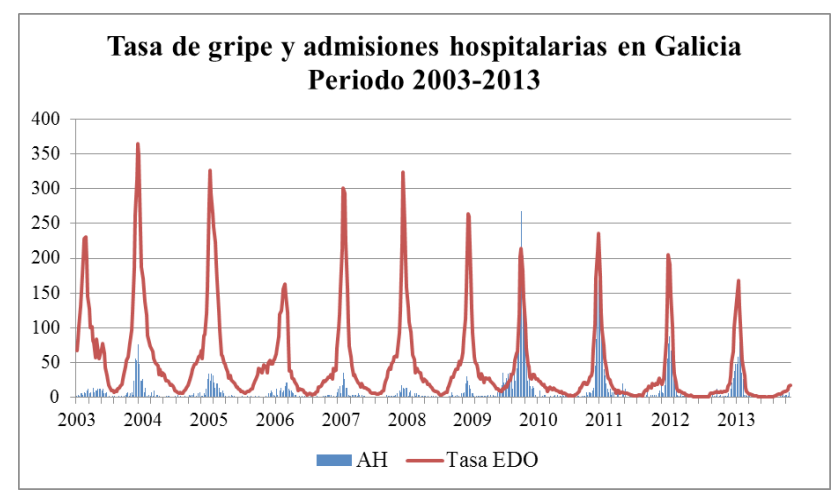

Fig. 1:Tasa de gripe y admisiones hospitalarias por semana epidemiológica en Galicia para el periodo 2003-2013

La Figura 1 muestra cómo el número de $\mathrm{AH}$ asociadas a la gripe experimenta un aumento muy relevante en los últimos años de la serie respecto a años precedentes. La alerta social generada en base a la gripe A (H1N1) del año epidémico 2009-10 puede explicar el extraordinario incremento de las admisiones hospitalarias, no de tasa gripal, que tienen lugar ese año.

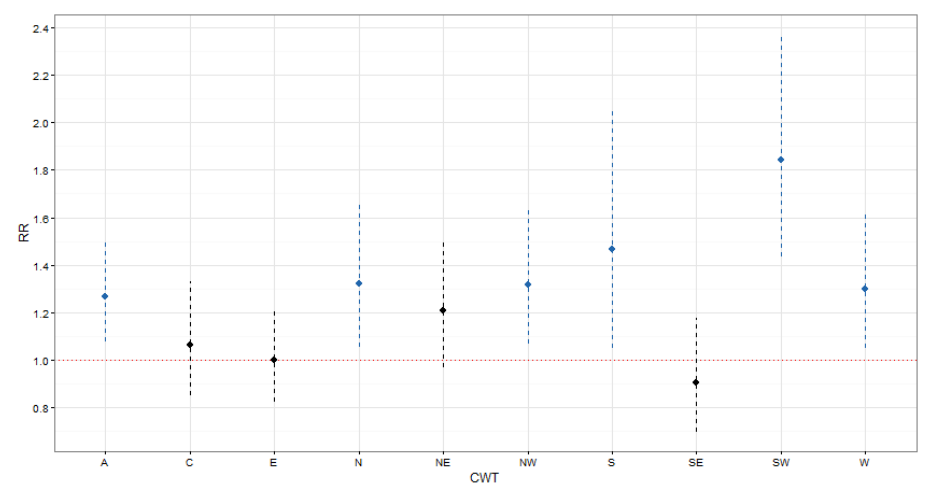

Fig. 2: Efectos de los CWT sobre los ingresos hospitalarios por gripe en el mismo día en la Comunidad Autónoma de Galicia. En color azul los RR significativos.

Los resultados obtenidos para los RR muestran importantes efectos significativos en la modelización de los RR con situaciones atmosféricas del SW $(1,84[1,43-2,36])$, $\mathrm{S}(1,46[1,04-2,04]), \mathrm{W}(1,3$ [1,04-1,61]), NW $(1,31$ [1,06-1,62]), N (1,32 [1,05-1,65]) y A $(1,26[1,07-1,49])$ (Fig. 2). El aumento más grande del riesgo de ingreso hospitalario, con un $84 \%$, se produce con una circulación del Suroeste. Las situaciones atmosféricas del S, SW, A y $\mathrm{C}$ han mostrado efectos significativos en todos los niveles de retardo (Fig. 3). Los mayores aumentos de riesgo de hospitalización por gripe llegan 
a ser del $86 \%$ y el $71 \%$ con una situación SW y un retardo de 5 y 6 días, respectivamente. La estructura de los efectos retardados se caracteriza por un patrón temporal irregular.

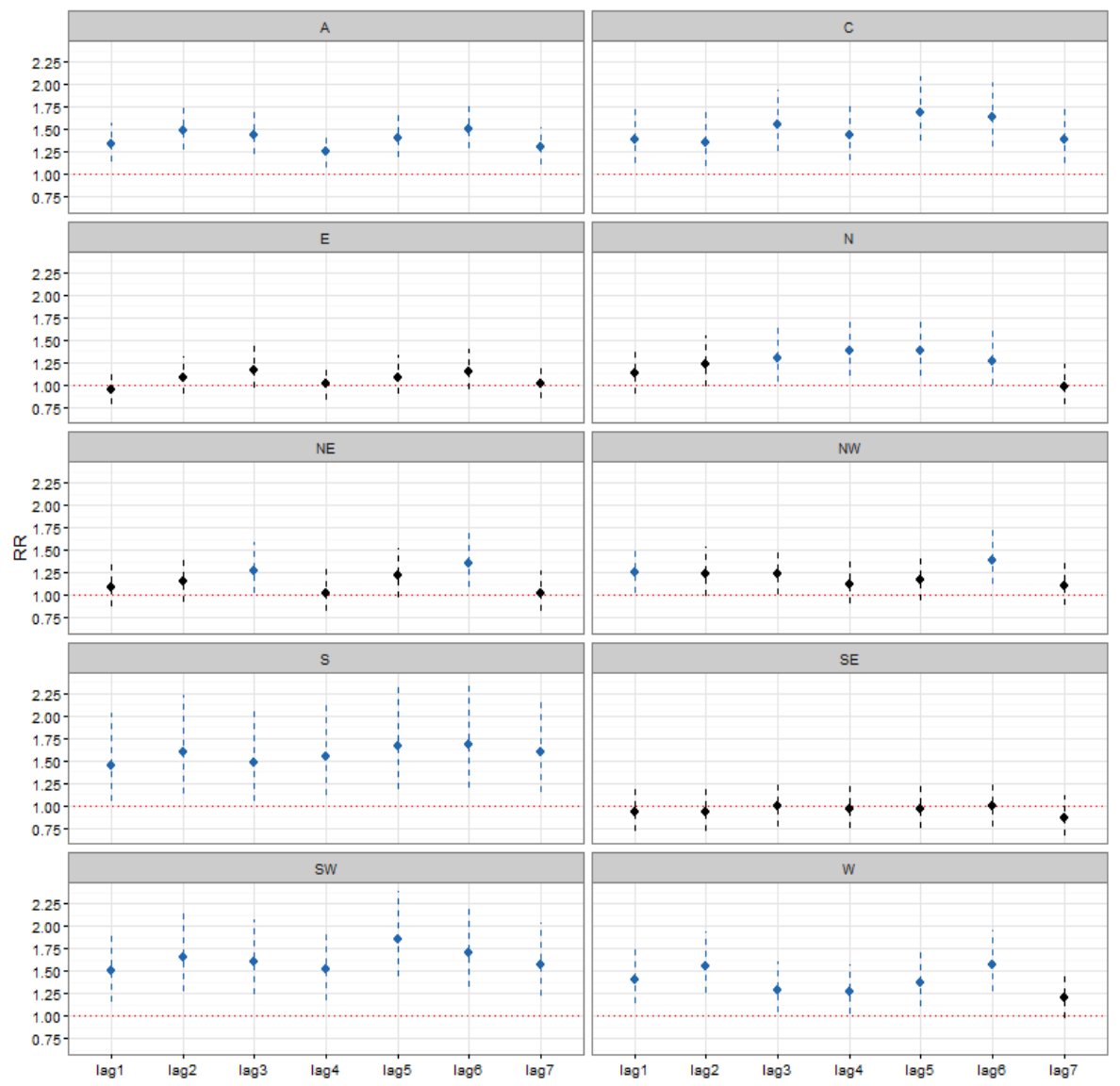

Fig. 3: Efectos retardados de los CWT (1-7 dias) sobre los ingresos hospitalarios por gripe en la Comunidad Autónoma de Galicia. En color azul los RR significativos.

No obstante destacan, por un lado, un primer pico en el segundo y/o tercer día, pero sobre todo un segundo pico más acusado en el quinto y/o sexto día después de los tipos de circulación mencionados, especialmente con las situaciones del SW y del $\mathrm{S}$ que provocan ambas unos riesgos relativos superiores al $70 \%$. Situaciones que en invierno dan lugar a unos tipos de tiempo lluviosos, con temperaturas suaves y con un muy alto grado de humedad relativa y absoluta, pues las masas de aire que llegan hasta Galicia proceden de latitudes subtropicales del Atlántico. Véase también cómo, en el otro extremo, los tipos de circulación del $1^{\circ}$ y $2^{\circ}$ cuadrantes (NE, E, SE), que dejan unos tipos de tiempo secos y fríos, no presentan a apenas ningún riesgo relativo significativo los diferentes niveles de retardo. 
Durante las 10 semanas de mayor número de admisiones hospitalarias hubo un claro predominio de situaciones de tercer y cuarto cuadrante especialmente de SW y W. Tres de estas semanas fueron las más significativas por número de ingresos con 660 hospitalizaciones acumuladas $(188+264+204)$ que tuvieron lugar de forma secuencial desde la última semana de octubre a mediados de noviembre de 2009. Los meses de septiembre y octubre de este año fueron muy secos en Galicia, con un predominio de situaciones $\mathrm{A}, \mathrm{E}$ y $\mathrm{NE}\left(1^{\circ}\right.$ y $2^{\circ}$ cuadrantes $)$ que generan un tiempo con ausencia de precipitaciones, una muy baja humedad. Estas condiciones son teóricamente favorables para la supervivencia del virus y su transmisión (Lowen et al. 2007, Polozov et al. 2008, Shaman \& Kohn 2009, Tamerius et al. 2011). Tras el 19 de octubre comienza una larga racha de situaciones del SW, W y NW $\left(3^{\circ}\right.$ y $4^{\circ}$ cuadrantes) que cambiaron el tiempo, con un incremento considerable de la humedad (de valores del $30-40 \%$ pasamos a $80-90 \%$ ), y un descenso brusco de las temperaturas (de ta máx entre 20 y $25^{\circ}$, esa semana pasamos a máximas por debajo de los $15^{\circ}$ y acompañadas de precipitación. Una secuencia de días lluviosos y húmedos y fríos prolongada favorecen el fenómeno epidemiológico del "crowding" o concentración de individuos en lugares cerrados y la probabilidad de difusión del virus de la gripe aumenta considerablemente y en consecuencia los ingresos hospitalarios asociados a la influenza. Ocurre algo similar en los días previos a los mayores incrementos de EDO. De los 10 mayores incrementos semanales de EDO de la serie, en 5 de ellos hay de nuevo un predominio de situaciones del $3^{\circ}$ y $4^{\circ}$ cuadrantes (SW, W, NW y S). Hay que destacar también que en estos mismos casos, con anterioridad a las situaciones del $\mathrm{W}$, tuvieron lugar secuencias de situaciones anticiclónicas y del E que dieron lugar a un tiempo estable, con valores de humedad mínimos en torno al 30/40\%, y con heladas nocturnas. También es cierto que en otras 5 ocasiones no sucede así lo que pone de manifiesto la complejidad de la expansión de enfermedades infecciosas como la influenza y de su relación con factores meteorológicos.

\section{CONCLUSIONES}

Los aumentos y descensos semanales de la tasas de gripe se encuentran relacionados con las admisiones hospitalarias La modelización entre el número de ingresos hospitalarios por gripe y los tipos de circulación atmosférica muestra que se producen importantes efectos significativos de dichos tipos sobre la variable de exposición. Los riesgos relativos (RR) más elevados tienen lugar con situaciones atmosféricas del SW y del S, seguidas de los tipos del W, NW, N y A. Los mayores aumentos del riesgo de hospitalización por gripe, superando el $70 \%$, se producen entre 5 y 6 días después de la llegada de masas de aire procedentes del SW y del S, que propician en buena parte del territorio gallego unos tipos de tiempo lluviosos, con temperaturas suaves y con unos altos grados de humedad relativa y absoluta. Estos retardos son teóricamente aceptables para explicar el periodo medio de incubación que transcurre entre el momento de la infección y la aparición de los síntomas siempre dependiendo del tipo de virus

Las situaciones del SW, W, NW o S son predominantes antes de los días con mayores incrementos de EDO o de los valores de ingresos hospitalarios más altos de la serie. Con anterioridad a estas situaciones del W, tuvieron lugar secuencias de situaciones anticiclónicas y del E que dieron lugar a un tiempo estable, seco y frío. Este 
proceso coincide con la hipótesis de trabajo planteada si bien ocurre así en la mitad de los episodios de gripe analizados lo que muestra nuevamente la dificultad para encontrar relaciones directas en proceso epidemiológicos que funcionan de modo independiente cada año. Finalmente, algunos aspectos sociales y culturales, como las alertar mediáticas, pueden propiciar el aumento de las admisiones hospitalarias asociadas a la gripe independientemente del tipo de circulación dominante.

\section{AGRADECIMIENTOS}

Al Centro Nacional de Epidemiología. Instituto de Salud Carlos III. Sistema de Vigilancia de Gripe en España. Red Nacional de Vigilancia Epidemiológica. 2003-13. España. Agradecimientos a la Subdirección General de Proyectos de Investigación del Ministerio de Economía y Competitividad por la financiación recibida a través del proyecto de referencia CSO2013-46153-R y de título Aproximación biometeorológica al estudio de la variabilidad espacial de la gripe en la Península Ibérica. También a la Dirección General de Salud Pública, Calidad e Innovación del Ministerio de Sanidad, Servicios Sociales e Igualdad por facilitar el acceso al Registro de Altas Hospitalarias CMBD.

\section{REFERENCIAS}

Analitis, A., Katsouyanni, K., Biggeri, A., Baccini, M., Forsberg, B., Bisanti, L., Kirchmayer, U., Ballester, F., Cadum, E., Goodman, P.G., Hojs, A., Sunyer, J., Tiittanen, P., Michelozzi, P. (2008). Effects of cold weather on mortality: results from $15 \mathrm{eu}-$ ropean cities within the PHEWE project. Am J Epidemiol, 168, 1397-1408.

Bloom-Feshbach K, Alonso WJ, Charu V, Tamerius J, Simonsen L, et al. (2013) Latitudinal Variations in Seasonal Activity of Influenza and Respiratory Syncytial Virus (RSV): A Global Comparative Review. PLoS ONE 8(2): e54445. doi:10.1371/journal.pone.0054445

Donna Bower, Glenn R. McGregor, David M. Hannah and Scott C. Sheridan (2007). Development of a spatial synoptic classification scheme forWestern Europe. Int. J. Climatol. 27: 2017-2040. DOI: 10.1002/joc.1501

Fdez.-Arroyabe, P. (2002). "The develop and application of dibreakib method in order to study the relationship between atmospheric dynamic and flu rate in the city of Vitoria in the expansion period of the virus". ICB 2002. AMS p.31-33.

Fdez.-Arroyabe, P. (2004): Climate weather and flu diagnoses incidence in the region of Santander during the 1999-200 epidemic diffusion period. Aerobiologia 20, p. 223-228 Kluwer Academic Publishers

Fdez-Arroyabe, P. (2012) Influenza epidemics and Spanish climatic domains. Health, 4, 941-945. doi: 10.4236/health.2012.430144.

Hastie, T.J., Tibshirani, R.J. (1990) A generalized additive models. Volume 43 of Monographson Statistics \& Applied Probability. Chapman\&Hall/CRC.

Jenkinson, A.F. and Collinson, P. (1977). An initial climatology of Gales over the North Sea. Sinoptic Climatology Branch. Memorandum No 62. Meteorological Office. London 18. 
Jensen M.M. (1964) Inactivation of airborne viruses by ultraviolet irradiation. Appl Microbiol 12:418-420.

Jones PD, Hulme M, Briffa KR. (1993). A comparison of Lamb circulation types with an objective calssigication scheme. International Journal of Climatology, 13, 655-663.

Kalkstein, L., Sheridan S. (2004). A bioclimatological analysis of heat health: progress in heatmatch warning systems technology. El clima entre el Mar y la montaña. IV Congreso de la AEC Conferencias invitadas). Publicaciones de la AEC. SerieB, n ${ }^{\circ} 4$.

Lorenzo, M. N. Taboada J.J. and Gimeno L. (2008). Links between circulation weather types and teleconnection patterns and their influence on precipitation patterns in Galicia (NW Spain).International Journal of Climatology, 28, 1493-1505.

Lowen, A. C., Mubareka, S., Tumpey, T.M., García Sastre, A., J., Palese, P. (2006). The guinea pig as a transmission model for human viruses. Proceedings of the National Academy of Science - PNAS, vol 103, n 26, USA

Lowen AC, Mubareka S, Steel J, Palese P. (2007). Influenza virus transmission is dependent on relative humidity and temperature. PLoS Pathog 3:1470-1476.

Moser M, Bender T, Margolis H, Noble G. (1979). An outbreak of influenza aboard a commercial airliner. Am J Epidemiol 110(1):1-6.

Mossong J, Hens N, Jit M, Beutels P, Auranen K, Mikolajczyk R, et al. 2008. Social contacts and mixing patterns relevant to the spread of infectious diseases. PLoS Med 5(3):e74; doi:10. 1371/journal.pmed.0050074 [Online 25 March 2008].

Maclure, M., 1991. The case-crossover design: a method for studying transient effects on the risk of acute events. Am J Epidemiol, 133, 144-153.

Peng, R.D., Dominici, F., 2008. Statistical methods for environmental epidemiology711 withR.Acasestudyinairpollutionandhealth. ¡UseR!,Springer.

Rasilla, D. (2000). Synoptic scale circulation conditions over the Iberian peninsula during extreme NAO phases. IGU Chapman Conference on NAO, Orense (Spain).

Royé, D., Taboada, J., Martí, A. y Lorenzo, N. (2016): Winter circulation weather and hospital admissions for respiratory disease in Galicia (Spain). Int. Journal of Biometeorology, Vol. 60, Issue 4, 507-520.

Sáez, M., Cadarso-Suárez, C., Figueiras, A., 2003. Np.or: an s-plus function for pointwise non parametric estimation of odds-ratios of continuous predictors. Comput Methods Programs Biomed, 71,175-179.

Schaffer FL, Soergel ME, Straube DC. 1976. Survival of airborne influenza virus: effects of propagating host, relative humidity, and composition of spray fluids. Arch Virol 51:263-273.

Schwartz B, Bell DM, Hughes JM. (1997) Preventing the emergence of antimicrobial resistance: a call for action by clinicians, public health officials, and patients [editorial]. JAMA. 1997;278:944-5

Shaman J, Kohn M. 2009. Absolute humidity modulates influenza survival, transmission, and seasonality. Proc Natl Acad Sci USA 106:3243-3248.

Tamerius J.D, Shaman J, Alonso WJ, Bloom-Feshbach K, Uejio CK, et al. (2013) Environmental Predictors of Seasonal Influenza Epidemics across Temperate 
and Tropical Climates. PLoS Pathog 9(3): e1003194. doi:10.1371/journal. ppat. 1003194

Tamerius J, Nelson MI, Zhou SZ, Viboud C, Miller MA, Alonso WJ. (2010) Global influenza seasonality: reconciling patterns across temperate and tropical regions. Environ Health Perspect. 2011 Apr;119(4):439-45. doi: 10.1289/ehp.1002383. Epub 2010 Nov 19. Review

Trigo RM, Da Camara CC. (2000). Circulation weather types and their influence on the precipitation regime in Portugal. International Journal of Climatology, 20, 1559-1581.

Xavier Badia Llach, Montserrat Roset Gamisans, José M. Francés Tudel, Concha Álvarez Sanz, Carlos Rubio Terrés, Estudio de costes de la gripe, Atención Primaria, Volume 38, Issue 5, 2006, Pages 260-267, ISSN 0212-6567, http:// dx.doi.org/10.1157/13092984.

Ye, X., Wolff, R., Yu, W., Vaneckova,P., Pan, X., Tong, S., 2012. Ambient temperature and morbidity: a review of epidemiological evidence. Environ Health Perspect, $120,19-28$. 